\title{
Efficient assessment of efficacy in post-traumatic peripheral neuropathic pain patients: pregabalin in a randomized, placebo-controlled, crossover study
}

This article was published in the following Dove Press journal: Journal of Pain Research

26 July 2012

Number of times this article has been viewed

\section{Tim M Jenkins \\ Trevor S Smart \\ Frances Hackman \\ Carol Cooke \\ Keith KC Tan}

Clinical Research, Pfizer Worldwide Research and Development, Sandwich, Kent, UK
Correspondence: Keith KC Tan

Medlmmune Limited, Milstein Building, Granta Park, Cambridge CB2I 6GH, UK

Tel +44 I 223898198

Fax +44 I223 47I I472

Email tank@medimmune.com
Background: Detecting the efficacy of novel analgesic agents in neuropathic pain is challenging. There is a critical need for study designs with the desirable characteristics of assay sensitivity, low placebo response, reliable pain recordings, low cost, short duration of exposure to test drug and placebo, and relevant and recruitable population.

Methods: We designed a proof-of-concept, double-blind, randomized, placebo-controlled, crossover study in patients with post-traumatic peripheral neuropathic pain (PTNP) to evaluate whether such a study design had the potential to detect efficacious agents. Pregabalin, known to be efficacious in neuropathic pain, was used as the active analgesic. We also assessed physical activity throughout the study.

Results: Twenty-five adults (20-70 years of age) with PTNP for $\geq 3$ months entered a screening week and were then randomized to one of the two following treatment sequences: (1) pregabalin followed by placebo or (2) placebo followed by pregabalin. These 2-week treatment periods were separated by a 2 -week washout period. Patients on pregabalin treatment received escalating doses to a final dosage of $300 \mathrm{mg} /$ day (days 5-15). In an attempt to minimize placebo response, patients received placebo treatment during the screening week and the 2-week washout period. Average daily pain scores (primary endpoint) were significantly reduced for pregabalin versus placebo, with a mean treatment difference of -0.81 (95\% confidence interval: -1.45 to -0.17 ; $P=0.015)$.

Conclusion: The efficacy of pregabalin was similar to that identified in a large, parallel group trial in PTNP. Therefore, this efficient crossover study design has potential utility for future proof-of-concept studies in neuropathic pain.

Keywords: pregabalin, post-traumatic peripheral neuropathic pain, randomized crossover trial, placebo response, actigraphy

\section{Introduction}

Neuropathic pain $(\mathrm{NeP})$, caused by a lesion or disease affecting the somatosensory system, ${ }^{1}$ may be difficult to treat owing to its persistence, resistance to standard analgesics, and the involvement of multiple mechanisms in the peripheral and/or central nervous systems. Patients with NeP present with remarkable phenotypic heterogeneity across the major NeP syndromes. ${ }^{2}$ The variety of sensory abnormalities in those patients may, in part, explain the challenge of designing effective clinical trials with high sensitivity and specificity.

Traditionally, efficacy studies have focused on patients considered to represent relatively homogeneous NeP populations, such as postherpetic neuralgia (PHN) or painful diabetic peripheral neuropathy (DPN), and used parallel group study designs. 
However, recruitment of patients with PHN, who tend to be elderly, has become increasingly challenging because of greater uptake of the varicella-zoster vaccine ${ }^{3}$ and competing recruitment demands from clinical studies. Patients with DPN, although more easily recruited, tend to have relatively high rates of concomitant medical conditions, variable glycemic control, and multiple therapeutic interventions. The resultant polypharmacy renders patients with DPN a less than ideal NeP population in which to investigate a novel agent, as there may be limited information concerning safety and drug-drug interaction. ${ }^{4}$

A chronic NeP condition that has been less studied in pharmacological trials is post-traumatic peripheral neuropathic pain (PTNP) following damage to peripheral nerves due to accidental or surgical injury. Given the many possible circumstances giving rise to PTNP, overall prevalence estimates are lacking. However, estimates exist for certain etiologies; for instance, chronic post-surgical pain is common and such patients may be a good target population for proof-of-concept studies. The estimated incidence of chronic postoperative pain after some surgical procedures ranges from $5 \%-50 \%$ depending on the operative procedures. ${ }^{5}$

Variability observed in placebo response in numerous trials $^{6,7}$ has been another issue that challenges NeP studies. This has led to a propensity for large sample sizes in initial proof-of-concept/efficacy studies. To address these issues, a number of studies have used crossover designs in various NeP populations and have demonstrated some efficacy with smaller sample sizes. ${ }^{8-10}$ An analysis of study characteristics influencing trial outcomes and placebo response in heterogeneous clinical trials suggest that parallel group designs are associated with higher placebo response rates. ${ }^{11}$ However, there is a lack of published literature that allows the assessment of placebo response and assay sensitivity of crossover versus parallel group designs for the same active drug in the same population of patients with NeP. In this study, we examined whether a crossover study design, with the addition of placebo administration during screening and the washout period, minimizes placebo response and optimizes assay sensitivity to an active drug whose efficacy was established in a parallel group study.

The study assessed the utility of a daily time-locked pain score (using Actiwatch ${ }^{\circledR}$ Score $^{12}$ [Philips Respironics, Murrysville, PA]) in an attempt to improve reliability and reduce study costs. The Actiwatch also enabled an investigation of actigraphy as an exploratory endpoint. Actigraphy, an objective measure of physical activity, has been widely used to assess sleep and circadian rhythms. ${ }^{13,14}$
However, there has been limited assessment of the utility of actigraphy in pain studies. ${ }^{15-17}$

Pregabalin is a ligand of the $\alpha_{2} \delta$-subunit of voltagesensitive $\mathrm{Ca}^{2+}$-channels. ${ }^{18}$ Using parallel group study designs, the analgesic efficacy of pregabalin has been demonstrated in several NeP conditions including DPN, ${ }^{19-21} \mathrm{PHN},{ }^{19,22,23} \mathrm{NeP}$ associated with spinal cord injury, ${ }^{24}$ and recently PTNP. ${ }^{25}$ Therefore, pregabalin was selected as the active agent to assess the sensitivity of the current crossover study design.

\section{Methods}

\section{Study population}

Men or women aged 18-80 years with a diagnosis of PTNP, confirmed by a pain specialist and persistent for $\geq 3$ months following the traumatic event, were recruited at four sites: two in Canada and two in Sweden. Patients with NeP not as a result of trauma - trigeminal neuralgia, central pain (due to cerebrovascular lesions, multiple sclerosis, and/or traumatic spinal cord injuries, including spinal surgery), complex region pain syndrome type I, phantom limb pain, radiculopathy, DPN, or PHN - were excluded from this study. Patients with any other coexisting pain that could not be discriminated from PTNP, in the opinion of the patient or clinician, or who had depression or any other medical conditions that would impair their ability to participate in the study were excluded. Patients with creatinine clearance $\leq 60 \mathrm{~mL} /$ minute or a positive urine illicit drug screen were also excluded. Women who were breastfeeding or pregnant were excluded and women of childbearing potential were required to use reliable contraception. In addition, patients who had previously failed to respond to pregabalin ( $\geq 300 \mathrm{mg} /$ day), were intolerant to $\geq 300 \mathrm{mg}$ of pregabalin, or who had previously failed to respond to gabapentin ( $\geq 1800 \mathrm{mg} /$ day) were excluded from the study.

Randomization criteria required patients to have discontinued and washed out of prohibited medications, including medications to relieve PTNP (ie, antidepressants, anticonvulsants/antiepileptics, opioids, selective serotonin and dual reuptake inhibitors, long-acting benzodiazepines, muscle relaxants, and topical analgesics), for specific washout periods before initiating the screening week. Patients were required not to initiate or change ongoing nonpharmacological therapies (eg, acupuncture and transcutaneous electrical neural stimulation); however, regular daily use of medications to treat stable conditions was allowed, provided that the medication was not prohibited and was kept constant throughout the study. In addition, patients taking nonsteroidal anti-inflammatory drugs or cyclooxygenase- 2 inhibitors were 
permitted to remain on these for the duration of the study, provided the doses were stable and expected to remain so for the duration of the study. Stable low-dose codeine was the only exception to a prohibition on opioids, with a maximum daily dose of $32 \mathrm{mg}$ permitted and a maximum weekly dose not exceeding $128 \mathrm{mg}$, provided the doses were stable and expected to remain so for the duration of the study.

Pain was rated on an 11-point numeric rating scale $(0=$ no pain to $10=$ worst possible pain). Patients were required to have at least four daily pain scores over the 7-day screening period prior to randomization and to have an average score of $\geq 4$. To examine the potential heterogeneity of the recruited PTNP population, a full medical history and cause of trauma were recorded, along with responses at screening to the Douleur Neuropathique 4 (DN4) questionnaire, ${ }^{26}$ but these were not used as randomization criteria.

\section{Study design and treatment}

This was an international, multicenter, double-blind, randomized, placebo-controlled, crossover study. The trial is registered at ClinicalTrials.gov (identifier NCT00654940). Enrollment began on May 5, 2008 and the last patient's last visit was on February 10, 2009. The protocol was reviewed and approved by institutional review boards and the study was conducted in accordance with the Declaration of Helsinki, Good Clinical Practice guidelines, and local laws and regulations. All patients gave written informed consent prior to any study related procedures being conducted, including any necessary washout period required for prohibited medications. After the screening week, eligible patients were randomized to one of two treatment sequences - pregabalin administered twice daily (BID) followed by placebo or placebo followed by pregabalin BID - by means of a computer-generated, pseudorandom code (blocking by site to try and ensure the balance of sequences within a site). These 2-week treatment periods were separated by a 2 -week washout period (Figure 1). For the pregabalin treatment period, all patients began with pregabalin $75 \mathrm{mg}$ on the evening of day 1 , which increased to $150 \mathrm{mg}$ /day on days 2 and 3,75 mg in the morning and $150 \mathrm{mg}$ in the evening on day 4 , and $300 \mathrm{mg}$ /day on days $5-15$. In addition, patients received placebo treatment (single-blind) during the screening week and 2-week washout periods. For the screening period, washout period, and placebo treatment arm, all placebo capsules were matched to pregabalin.

\section{Efficacy assessments}

The primary efficacy endpoint was the mean pain score for each treatment period calculated from the daily pain scores

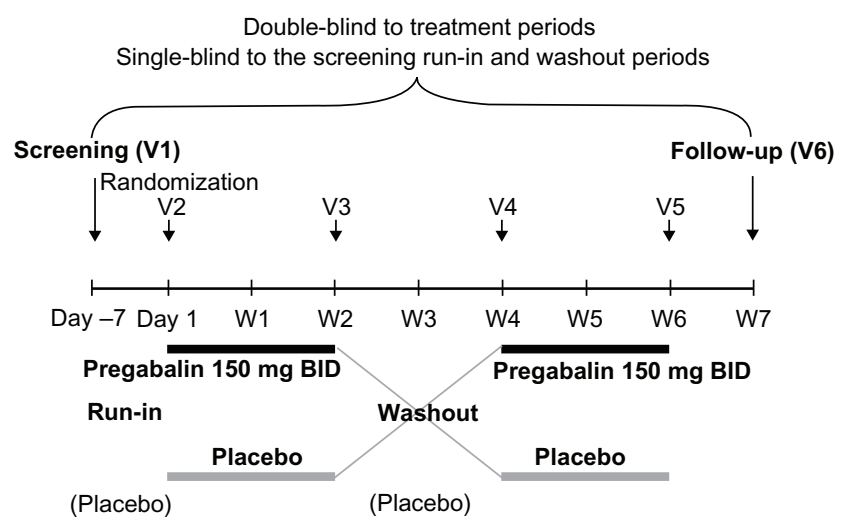

Figure I Schematic representation of the study design. Abbreviations: BID, twice a day; $\mathrm{V}$, visit; $\mathrm{W}$, week.

of the last 7 days of treatment, with a required minimum of at least four daily pain scores. Baseline pain score was defined as the mean of the last seven pretreatment pain scores. If less than seven pain scores were recorded at baseline, the available scores were used to determine the mean. If less than seven scores were recorded post-baseline, the available scores were used to determine the endpoint mean pain score. Pain over the last 24 hours was rated daily upon rising from bed. The Actiwatch Score device was worn on the wrist and pre-programmed to allow patient-initiated entry of daily pain scores on an 11-point numeric rating scale.

Two exploratory secondary endpoints were also included in this study. The Neuropathic Pain Symptom Inventory (NPSI) $^{27}$ questionnaire was completed on the following scheduled visits to the clinic: day 1 , week 2 , week 4 , and week 6 . The self-administered NPSI includes ten different pain symptom descriptors (eg, burning, stabbing, electric shock) and two temporal items, and allows for discrimination and quantification of five distinct clinically relevant dimensions of NeP. Actigraphy data were captured throughout the study using the Actiwatch Score, which contains an accelerometer that allows an objective measure of physical activity by monitoring body motion during sleep and waking hours. These devices were worn continuously throughout the study and the data related to total, average, and peak activity were downloaded during clinic visits. Actigraphy data were calculated as described previously. ${ }^{12}$ The values used in the analysis were the mean activity score for each treatment period calculated from the daily activity scores of the last 7 days of treatment.

\section{Tolerability and safety assessments}

All spontaneously reported and observed adverse events (AEs) were recorded at each clinic visit. Standard clinical 
laboratory tests, blood pressure and heart rate, triplicate electrocardiograms, and urine drug testing were conducted at screening. In addition, all female patients of childbearing potential were given a pregnancy test at screening. A general physical examination also was performed at both screening and follow-up.

\section{Data analysis}

The primary comparison was between pregabalin and placebo treatments. The study was designed to detect a difference in means (pregabalin-placebo) of at least -1.0 (negative numbers indicating superiority of pregabalin over placebo). A total of 16 patients completing the study would achieve at least $80 \%$ power for the primary comparison. Assuming a 30\% drop-out rate, the study had to randomize 24 patients. The primary analysis was based on the full-analysis set (FAS) population, and a mixed analysis of covariance (ANCOVA) model was fitted, accounting for the patient as a random effect, the period and treatment as fixed effects, and the baseline scores (for each treatment period) introduced as inter- and intrapatient covariates. The interpatient covariate was the patient's mean baseline values (the mean of each patient's period 1 and period 2 baseline values) and the intrapatient covariate was the difference between the patient's baseline measurement for the specific period and his/her mean baseline. Taking into account that patients may have recorded more than one response per day, an algorithm was applied to reduce the score to one pain score per day. The first daily score recorded after 4 am was chosen as the daily pain score in the study. The adjusted treatment difference (pregabalinplacebo) was reported together with the corresponding standard error (SE) and 95\% confidence interval (CI). For sensitivity, the primary analysis was repeated using a perprotocol (PP) population that included only those patients who completed the study with no major protocol deviations. NPSI was analyzed using a mixed effects ANCOVA and was based on the FAS population. The period and treatment were fitted as fixed effects, the patient was fitted as a random effect, and the baseline was fitted as two covariates. The treatment comparison was between pregabalin and placebo. The adjusted treatment difference (pregabalinplacebo) was reported together with the corresponding SE and $95 \%$ CI. Data were summarized for NPSI total score and the five clinically relevant dimensions of NeP. Patient activity was collected hourly for the following variables: peak, average, and total activity. These were then divided into day ( 8 am-8 pm), morning ( 8 am-2 pm), afternoon
( 2 pm-8 pm), and night ( $1 \mathrm{am}-5 \mathrm{am})$, and analyzed as for the primary endpoint.

\section{Results \\ Patients}

In total, 28 patients were screened, with 25 patients meeting the randomization criteria (Figure 2). One patient withdrew because of nausea and abdominal pain (during placebo treatment) and one patient withdrew consent during the washout between treatment periods. These two withdrawn patients and the 23 patients who completed the study comprised the FAS population. There were 20 patients, determined prior to unblinding, who completed the study with no major protocol deviations and were included in the PP population. In addition to the two patients who discontinued, one patient was $<80 \%$ compliant with medication, one patient had recorded excessive daily pain scores, and one patient had less than four pain scores during screening.

In total, 11 males (20-60 years of age) and 14 females (28-70 years of age) were randomized into the study and their baseline demographic and clinical characteristics, including screening pain intensities, were similar in the two randomized treatment sequences (Table 1). Surgery was the

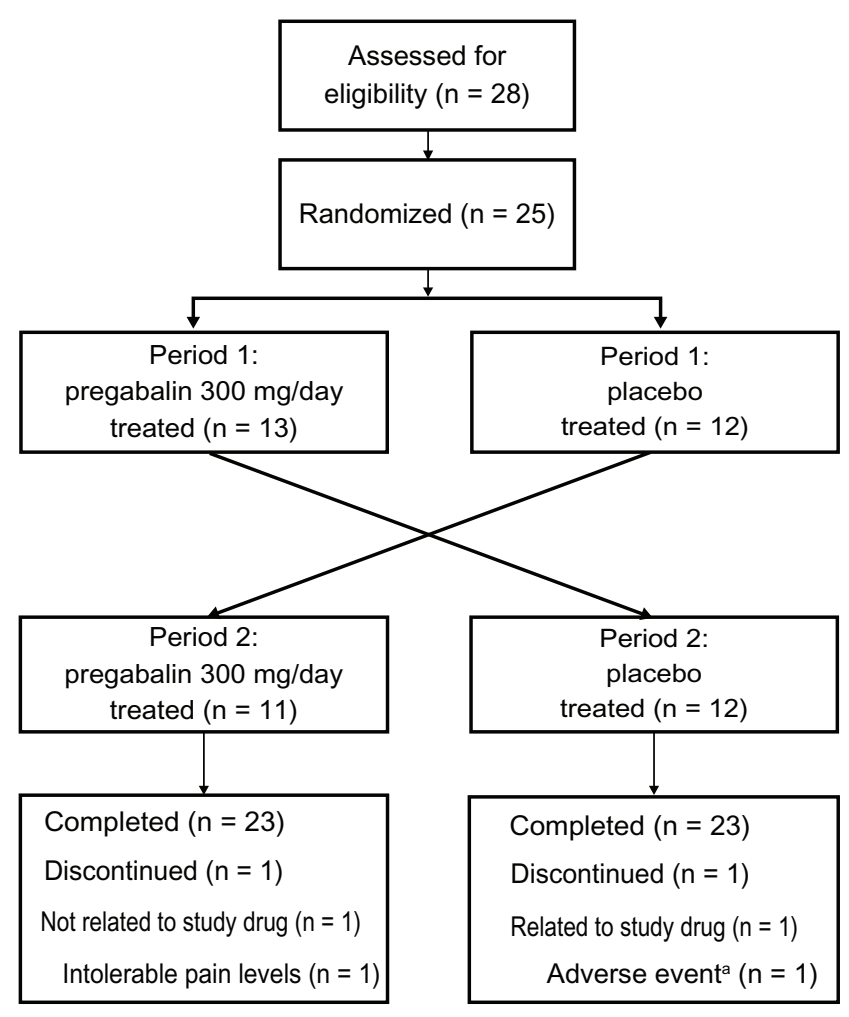

Figure 2 Patient disposition. Note: ${ }^{a}$ Nausea, abdominal pain. 
Table I Patient demographics

\begin{tabular}{|c|c|c|}
\hline Treatment sequence & $\begin{array}{l}\text { Pregabalin- } \\
\text { placebo } \\
(n=13)\end{array}$ & $\begin{array}{l}\text { Placebo- } \\
\text { pregabalin } \\
(\mathrm{n}=\mathrm{I})\end{array}$ \\
\hline \multicolumn{3}{|l|}{ Age (years) } \\
\hline Mean \pm SD & $50.2 \pm 16.7$ & $49.4 \pm 11.8$ \\
\hline Range & $20-70$ & $22-70$ \\
\hline Male (n [\%]) & $6(46.15)$ & $5(4 \mid .66)$ \\
\hline \multicolumn{3}{|l|}{ Race (n [\%]) } \\
\hline White & $13(100)$ & $12(100)$ \\
\hline \multicolumn{3}{|l|}{ Primary diagnosis MedDRA } \\
\hline \multicolumn{3}{|l|}{ (v I2.0) preferred term } \\
\hline Nerve injury & 13 & 12 \\
\hline Baseline pain score, mean \pm SD & $6.03 \pm 1.29$ & $5.96 \pm 0.88$ \\
\hline
\end{tabular}

assigned cause of PTNP for most patients $(n=19)$, followed by accident $(\mathrm{n}=3)$ and fracture $(\mathrm{n}=3)$.

\section{Primary efficacy endpoint}

The mean baseline pain score for period 1 was approximately 6.0 in both the pregabalin/placebo $(\mathrm{n}=13)$ and placebo/ pregabalin $(n=12)$ sequences, while the mean baseline pain score for period 2 was 5.3 in both sequences. Negligible decreases from baseline in mean pain scores were seen with placebo treatment (least squares mean [SE] values of 0.03 [0.216] for the FAS population and 0.14 [0.249] for the PP population). Pregabalin significantly reduced mean pain scores at the end of treatment compared with placebo for both FAS ( $P=0.0152$; Figure 3$)$ and PP $(P=0.0087)$ populations. At the end of treatment, the mean difference between pregabalin and placebo was -0.81 (95\% CI: -1.45 to -0.17$)$ for the FAS population and -1.07 (95\% CI: -1.84 to -0.31 ) for the PP population (Table 2). There was no evidence of any treatment carryover effect from period 1 when looking at the baseline pain scores in period 2. Accounting for the overall reduction in pain across the study (less pain in the second period than the first) by fitting a period effect showed

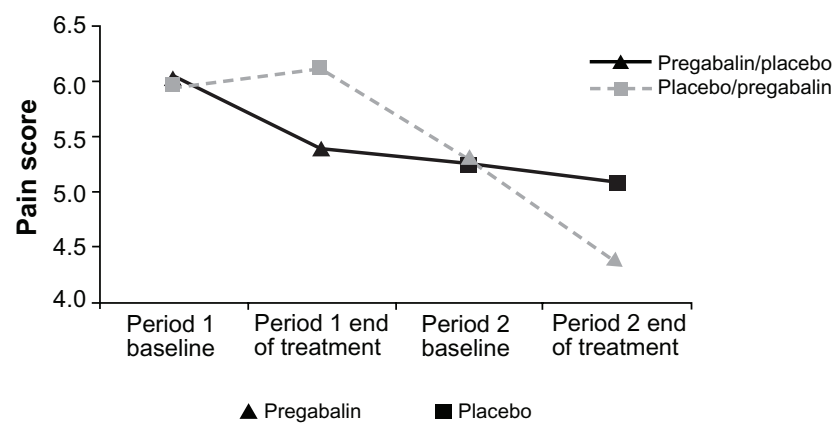

Figure 3 Mean baseline and endpoint pain scores by period and by treatment. that the treatment effect, pregabalin-placebo difference, was consistent across the sequences and periods, and there was no evidence of any treatment by period interaction or carryover.

\section{Exploratory secondary endpoints}

Actigraphy activity data were summarized by day $(8 \mathrm{am}-8 \mathrm{pm})$, morning ( $8 \mathrm{am}-2 \mathrm{pm})$, afternoon ( $2 \mathrm{pm}-8 \mathrm{pm})$, and night ( $1 \mathrm{am}-5 \mathrm{am})$. Average activity during the morning demonstrated the most apparent treatment effect with pregabalin, although this was not statistically significant at the two-sided $5 \%$ level, with a treatment difference of 44.2 (95\% CI: -4.48 to $92.92 ; P=0.07)$. This was equivalent to an improvement of approximately $10.5 \%$ over placebo. Of the 25 randomized patients with investigator-diagnosed PTNP, only one patient had less than four positive responses to the 10-item DN4 questionnaire, with this patient reporting positive responses to burning pain, tingling, and investigator-reported touch hypoesthesia. Patient-reported type of pain on the DN4 questionnaire included the following: burning (80\%), tingling (76\%), pins and needles (76\%), electric shock (68\%), numbness (64\%), itching (36\%), and painful cold (24\%). Investigator-reported induction of hyperalgesia included touch $(72 \%)$, pricking $(72 \%)$, or brushing $(48 \%)$. There was no significant difference between pregabalin and placebo on the NPSI total score or any of the five distinct clinically relevant dimensions of NPSI (data not shown).

\section{Tolerability and safety}

In total, $42 \%$ of patients reported treatment-emergent AEs during the placebo treatment periods and $46 \%$ of patients reported treatment-emergent AEs during the pregabalin treatment periods. All AEs were mild or moderate and there were no severe AEs. One patient withdrew owing to nausea and abdominal pain during a placebo treatment period. The most common treatment-emergent AEs were dizziness with six events reported during the pregabalin treatment periods and one event during the placebo treatment period; nausea with four events reported during the pregabalin treatment periods and three during the placebo treatment periods; and somnolence with three events reported during the pregabalin treatment periods.

\section{Discussion}

As novel agents are sought to address the high medical need presented by patients with $\mathrm{NeP}$, there is a concomitant requirement to develop robust study designs able to reliably detect efficacious agents. In addition, there is a 
Table 2 Mean pain score results at the end of treatment using the full-analysis set and the per-protocol set

\begin{tabular}{lllll}
\hline Population & Treatment & LS mean & Treatment difference (SE) & 95\% CI \\
\hline Full-analysis set & Pregabalin & 4.89 & $-0.81(0.305)^{*}$ & $(-1.45$ to -0.17$)$ \\
& Placebo & 5.70 & & \\
Per-protocol & Pregabalin & 4.68 & $-1.07(0.362)^{* *}$ & $(-1.84$ to $-0.3 \mathrm{I})$ \\
& Placebo & 5.75 & & \\
\hline
\end{tabular}

Notes: Primary endpoint: endpoint mean pain score - average of the last 7 days on treatment. Analysis of covariance on end of treatment, fixed effects of period and treatment, two baseline covariates, and random patient effect. $* P=0.0152 ; * * P=0.0087$ (two-sided).

Abbreviations: $\mathrm{Cl}$, confidence interval; LS, least squares; $\mathrm{SE}$, standard error.

need to identify an alternative NeP population for early proof-of-concept/efficacy studies other than the traditional PHN and DPN patient populations. Finally, the potential for variable placebo responses, whether in initial proof-ofconcept/efficacy studies or larger Phase III/IV trials, continues to be a challenging aspect of randomized controlled analgesic studies.

In this simple two-way crossover study with placebo administration during screening and washout periods and just 25 patients, we have efficiently detected a significant treatment effect with pregabalin in patients with PTNP. The reduction in pain intensity observed in this current study $(-0.81[95 \%$ CI: -1.45 to -0.17$])$ is similar to that obtained in a recently completed large PTNP parallel group-designed study $(-0.62$ [ $95 \%$ CI: -1.09 to -0.15 ]) comprising 254 patients, ${ }^{25}$ and is comparable with the efficacy signals seen in a variety of $\mathrm{NeP}$ populations. ${ }^{19-24}$ There was no evidence of carryover, meaning the effect of a treatment in the first period affecting the results of the second period. However, there was a reduction in pain over time throughout the study (Figure 3), which is typically observed in both parallel group and crossover pain studies. Notably, the reduction in pain between the end of period 1 and the baseline of period 2 was greater in the placebo-treated patients than in the pregabalin-treated patients. The reason for this observation is unclear. A possible explanation is that on placebo there was a general reduction in pain over time, but this reduction was greater for the patients on pregabalin. When the patients on pregabalin were switched to placebo during the washout period they returned towards the placebo time course and, hence, the reduction in pain after pregabalin was less than after placebo during the washout. This would be equivalent to a return to baseline if there was no reduction in pain on placebo. The lower baseline values in period 2 than period 1 were accounted for in the analysis by incorporating a period effect. If a future study lasts longer with more periods, then there could be a further reduction in pain, which reduces the window that a treatment effect can be seen. Care should be taken if extending the study design to three or more periods.
The crossover study design presented here is relatively straightforward, without the need for patient enrichment strategies, extensive screening, or run-in phases. Therefore, this study design facilitates enrollment of a small number of patients and had a minimal withdrawal rate. This is in contrast to the enriched-enrollment randomized withdrawal design that requires enrollment of a larger number of patients to the titration period, with only a small number of them entering randomization. ${ }^{28}$

In order to assess the maximal sensitivity of the current study and to maximize subject retention, we excluded patients who had previously failed to respond to pregabalin or gabapentin or were intolerant to a low dose of pregabalin. An advantage of this approach is that failure to detect efficacy would indicate lack of utility of the study design clearly. A disadvantage is that the generalizability of these results may be limited because of a potential selection bias from the exclusion criterion.

Recruitment rates from the four sites demonstrated that future proof-of-concept/efficacy studies could be readily supported using this same general design and patient population, and it represents a potential reduction in the number of sites generally required to support similar numbers of patients with PHN or DPN. Additionally, the response on the DN4 questionnaire indicated that the patients suffered from NeP and that the DN4 is a reliable tool for the inclusion of patients with PTNP in future studies.

Perhaps the most striking result, after the clear demonstration of a statistically significant treatment effect with pregabalin, was the very low placebo response observed with this particular study design. In recent years, there has been an increase in the number of chronic pain clinical trials, in which the treatment being evaluated did not differ significantly from placebo in the primary efficacy measure. ${ }^{29}$ It was suggested that it was due to excessive response rates in the placebo groups.$^{29}$ As indicated previously, in seeking to identify a study design that more efficiently evaluated the potential efficacy of novel analgesic agents in NeP, we sought to introduce elements that might diminish the 
placebo response. Therefore, patients received matching placebo during screening and during the two washout periods. We observed minimal changes from baseline in mean pain score during placebo treatment in each treatment period, which resulted in an overall low placebo response. With this design, the patients know that they will receive both treatments but are blinded to the specific time when treatments are changed; therefore, this may help overcome the patients' expectation that leads to placebo responses. ${ }^{30-32}$ It is difficult to determine with certainty whether the addition of placebo during baseline and washout contributed to the minimal placebo response. Directly addressing this question would require randomization to a parallel study group that did not receive placebo during the baseline and washout period, which would significantly increase the complexity of the investigation. The current study provided a pragmatic assessment of a placebo reduction strategy.

We have attempted to identify selective or preferential effects of pregabalin on the different dimensions of NeP based on responses of the NPSI questionnaire, but found no significant difference between pregabalin and placebo on the NPSI total score or any of the five distinct clinically relevant dimensions of NPSI. Larger studies would be required to confirm any differential effects of pregabalin on NeP symptom dimensions.

In this study, we investigated the utility of the Actiwatch Score in collecting patients' daily pain score. The first pain score entered after 4 am was taken as the daily pain rating over the last 24 hours (upon rising from bed) for all patients. Therefore, the use of the Actiwatch Score device or a similar device to record daily pain scores, in a time-related manner, appears to be practical, and in such small studies may provide a clear advantage over the set-up costs of other systems, such as automated phone services. Furthermore, when patients with chronic pain were evaluated for compliance by using a paper diary instrumented to track actual diary use, it was found that although patients submitted diaries indicating $90 \%$ compliance, the electronic records indicated that actual compliance was only $11 \%$, suggesting a high level of faked compliance. ${ }^{33}$ However, the study showed that there was high compliance with electronic diaries with enhanced compliance features..$^{33}$ The poor compliance or even faked compliance with paper diaries has important implications for the validity of the data. The Actiwatch Score device is a convenient system to collect pain ratings and may reduce the inaccuracies associated with ratings that rely on summaries of daily experiences in paper diaries based on memory. ${ }^{34}$
The Actiwatch Score device not only captured daily pain scores, but also enabled the exploratory secondary analysis of actigraphy data. None of the components demonstrated a significant treatment effect with pregabalin; however, the largest treatment effect was seen with average activity in the morning, with an improvement over placebo of approximately $10.5 \%$. One study in patients with $\mathrm{NeP}^{20}$ reported an increase in daytime activity (assessed via actigraphy) with a reduction in pain scores; however, as this was not a blinded study, it was difficult to interpret these results. Although the actigraphy results in our study were not significant, they indicated that it is worth further investigation. A larger study will be required to confirm the validity of these results.

In conclusion, this study describes a two-way crossover design that allowed efficient assessment of the efficacy of the active treatment, pregabalin, in a relatively small PTNP patient population. There was a fairly low placebo response, which may be related to the utilization of placebo during both the screening/run-in and washout phases between the treatment periods of this crossover design. In addition, the utility of the Actiwatch Score device to capture pain scores has been confirmed, and there is an indication that future studies should explore activity as a relevant endpoint in NeP. Finally, the results described here indicate that similar study designs of patients with PTNP, an alternative NeP population to patients with PHN and DPN, may represent a more efficient way to evaluate the potential efficacy of novel analgesic agents in future proof-of-concept/efficacy studies.

\section{Acknowledgments}

We would like to thank the co-investigators Dr Michael O’Mahony (Sarnia, Canada), Dr Jan Sorensen (Linkoping, Sweden), Dr Mats Persson (Jonkoping, Sweden), and Dr Cory Toth (Calgary, Canada).

\section{Disclosure}

This study was funded by Pfizer Inc. Tim Jenkins, Trevor Smart, Frances Hackman, Carol Cooke, and Keith Tan were employed by Pfizer Inc, during the design, execution, and interpretation of this study. Tim Jenkins is currently an employee of Eisai Ltd. Medical writing support was provided by Vardit Dror, PhD, of UBC Scientific Solutions and funded by Pfizer Inc.

\section{References}

1. Treede RD, Jensen TS, Campbell JN, et al. Neuropathic pain: redefinition and a grading system for clinical and research purposes. Neurology. 2008;70(18):1630-1635. 
2. Maier C, Baron R, Tolle TR, et al. Quantitative sensory testing in the German Research Network on Neuropathic Pain (DFNS): somatosensory abnormalities in 1236 patients with different neuropathic pain syndromes. Pain. 2010;150(3):439-450.

3. Oxman MN, Levin MJ, Johnson GR, et al; Shingles Prevention Study Group. A vaccine to prevent herpes zoster and postherpetic neuralgia in older adults. N Engl J Med. 2005;352(22):2271-2284.

4. Triplitt C. Drug interactions of medications commonly used in diabetes. Diabetes Spectrum. 2006;19:202-211.

5. Akkaya T, Ozkan D. Chronic post-surgical pain. Agri. 2009;21(1):1-9.

6. Vase L, Riley JL 3rd, Price DD. A comparison of placebo effects in clinical analgesic trials versus studies of placebo analgesia. Pain. 2002; 99(3):443-452.

7. Irizarry MC, Webb DJ, Ali Z, et al. Predictors of placebo response in pooled lamotrigine neuropathic pain clinical trials. Clin J Pain. 2009; 25(6):469-476.

8. Gordh TE, Stubhaug A, Jensen TS, et al. Gabapentin in traumatic nerve injury pain: a randomized, double-blind, placebo-controlled, cross-over, multi-center study. Pain. 2008;138(2):255-266.

9. Ho TW, Backonja M, Ma J, Leibensperger H, Froman S, Polydefkis M. Efficient assessment of neuropathic pain drugs in patients with small fiber sensory neuropathies. Pain. 2009;141(1-2):19-24.

10. Yuan RY, Sheu JJ, Yu JM, et al. Botulinum toxin for diabetic neuropathic pain: a randomized double-blind crossover trial. Neurology. 2009;72(17): 1473-1478.

11. Katz J, Finnerup NB, Dworkin RH. Clinical trial outcome in neuropathic pain: relationship to study characteristics. Neurology. 2008;70(4): 263-272.

12. Gironda RJ, Lloyd J, Clark ME, Walker RL. Preliminary evaluation of reliability and criterion validity of Actiwatch-Score. J Rehabil Res Dev. 2007;44(2):223-230.

13. Ancoli-Israel S, Cole R, Alessi C, Chambers M, Moorcroft W, Pollak CP. The role of actigraphy in the study of sleep and circadian rhythms. Sleep. 2003;26(3):342-392.

14. Lichstein KL, Stone KC, Donaldson J, et al. Actigraphy validation with insomnia. Sleep. 2006;29(2):232-239.

15. Agarwal S, Polydefkis M, Block B, Haythornthwaite J, Raja SN. Transdermal fentanyl reduces pain and improves functional activity in neuropathic pain states. Pain Med. 2007;8(7):554-562.

16. Kop WJ, Lyden A, Berlin AA, et al. Ambulatory monitoring of physical activity and symptoms in fibromyalgia and chronic fatigue syndrome. Arthritis Rheum. 2005;52(1):296-303.

17. Liszka-Hackzell JJ, Martin DP. An analysis of the relationship between activity and pain in chronic and acute low back pain. Anesth Analg. 2004;99(2):477-481.

18. Taylor CP. Mechanisms of analgesia by gabapentin and pregabalin calcium channel alpha2-delta [Cavalpha2-delta] ligands. Pain. 2009; 142(1-2):13-16.

19. Freynhagen R, Strojek K, Griesing T, Whalen E, Balkenohl M. Efficacy of pregabalin in neuropathic pain evaluated in a 12-week, randomised, double-blind, multicentre, placebo-controlled trial of flexible- and fixed-dose regimens. Pain. 2005;115(3):254-263.
20. Lesser H, Sharma U, LaMoreaux L, Poole RM. Pregabalin relieves symptoms of painful diabetic neuropathy: a randomized controlled trial. Neurology. 2004;63(11):2104-2110.

21. Rosenstock J, Tuchman M, LaMoreaux L, Sharma U. Pregabalin for the treatment of painful diabetic peripheral neuropathy: a double-blind, placebo-controlled trial. Pain. 2004;110(3):628-638.

22. Dworkin RH, Corbin AE, Young JP Jr, et al. Pregabalin for the treatment of postherpetic neuralgia: a randomized, placebo-controlled trial. Neurology. 2003;60(8):1274-1283.

23. Sabatowski R, Galvez R, Cherry DA, et al; 1008-045 Study Group. Pregabalin reduces pain and improves sleep and mood disturbances in patients with post-herpetic neuralgia: results of a randomised, placebocontrolled clinical trial. Pain. 2004;109(1-2):26-35.

24. Siddall PJ, Cousins MJ, Otte A, Griesing T, Chambers R, Murphy TK. Pregabalin in central neuropathic pain associated with spinal cord injury: a placebo-controlled trial. Neurology. 2006;67(10):1792-1800.

25. van Seventer R, Bach FW, Toth CC, et al. Pregabalin in the treatment of post-traumatic peripheral neuropathic pain: a randomized double-blind trial. Eur J Neurol. 2010;17(8):1082-1089.

26. Bouhassira D, Attal N, Alchaar H, et al. Comparison of pain syndromes associated with nervous or somatic lesions and development of a new neuropathic pain diagnostic questionnaire (DN4). Pain. 2005;114(1-2): 29-36.

27. Bouhassira D, Attal N, Fermanian J, et al. Development and validation of the Neuropathic Pain Symptom Inventory. Pain. 2004;108(3): 248-257.

28. Hewitt DJ, Ho TW, Galer B, et al. Impact of responder definition on the enriched enrollment randomized withdrawal trial design for establishing proof of concept in neuropathic pain. Pain. 2011;152(3):514-521.

29. Dworkin RH, Turk DC, Peirce-Sandner S, et al. Research design considerations for confirmatory chronic pain clinical trials: IMMPACT recommendations. Pain. 2010;149(2):177-193.

30. Benedetti F, Pollo A, Lopiano L, Lanotte M, Vighetti S, Rainero I. Conscious expectation and unconscious conditioning in analgesic, motor, and hormonal placebo/nocebo responses. J Neurosci. 2003;23(10): 4315-4323.

31. Quessy SN, Rowbotham MC. Placebo response in neuropathic pain trials. Pain. 2008;138(3):479-483.

32. Turner JA, Deyo RA, Loeser JD, Von Korff M, Fordyce WE. The importance of placebo effects in pain treatment and research. JAMA. 1994;271(20):1609-1614.

33. Stone AA, Shiffman S, Schwartz JE, Broderick JE, Hufford MR. Patient compliance with paper and electronic diaries. Control Clin Trials. 2003; 24(2):182-199.

34. Stone AA, Broderick JE, Schwartz JE, Shiffman S, Litcher-Kelly L, Calvanese P. Intensive momentary reporting of pain with an electronic diary: reactivity, compliance, and patient satisfaction. Pain. 2003;104(1-2): 343-351.
Journal of Pain Research

\section{Publish your work in this journal}

The Journal of Pain Research is an international, peer-reviewed, open access, online journal that welcomes laboratory and clinical findings in the fields of pain research and the prevention and management of pain. Original research, reviews, symposium reports, hypothesis formation and commentaries are all considered for publication.

\section{Dovepress}

The manuscript management system is completely online and includes a very quick and fair peer-review system, which is all easy to use. Visit http://www.dovepress.com/testimonials.php to read real quotes from published authors. 